\title{
Optimierung der kommunalen Gesundheitsversorgung: Erste Erfahrungen des Kommunalbüros für ärztliche Versorgung des Landes Bayern
}

\author{
Optimization of Community Health Care: First Experiences of the \\ Office for Medical Care in Districts and Municipalities of Bavaria
}

\section{(c) (1) $\circledast$}

\author{
Autoren \\ Gunnar Geuter, Thomas Ewert, Timo Deiters, Alfons Hollederer \\ Institut \\ Bayerisches Landesamt für Gesundheit und Lebensmittelsicherheit \\ (LGL), Sachgebiet Versorgungsqualität, Gesundheitsökonomie, \\ Gesundheitssystemanalyse (GE 6), Nürnberg
}

\author{
Schlüsselwörter \\ Vertragsärztliche Versorgung, Kommune, Öffentlicher Gesundheits- \\ dienst
}

Key words

service by panel doctors, municipality, public health service

Bibliografie

DOI http://dx.doi.org/10.1055/s-0042-102343

Online-Publikation: 18.3.2016 | Gesundheitswesen 2017; 79: 28-34

(c) Georg Thieme Verlag KG Stuttgart · New York

ISSN 0941-3790

\section{Korrespondenzadresse}

Gunnar Geuter, MPH

Bayerisches Landesamt für Gesundheit und Lebensmittelsicherheit (LGL)

Sachgebiet Versorgungsqualität, Gesundheitsökonomie,

Gesundheitssystemanalyse (GE 6)

Bayerisches Haus der Gesundheit

Schweinauer Hauptstraße 80

90441 Nürnberg

gunnar.geuter@|gl.bayern.de

\section{ZUSAMMENFASSUNG}

Hintergrund Die Auswirkungen des demografischen Wandels stellen große Herausforderungen an das Gesundheitswesen. Um die wohnortnahe Gesundheitsversorgung auch zukünftig zu erhalten, wird vor diesem Hintergrund die vertragsärztliche Versorgung zunehmend zu einem Handlungsfeld auf Landes- und kommunaler Ebene. Viele Kommunen befassen sich allerdings erstmals intensiv mit der Thematik und stehen dabei vor großen Herausforderungen. Denn es mangelt an Beratungs- und Unterstützungsangeboten. Ziel der Arbeit ist, die Konzeption eines neu eingerichteten Kommunalbüros für ärztliche Versorgung und dessen ersten Ergebnisse darzustellen.

Methodik In Bayern wurde vom Bayerischen Staatsministerium für Gesundheit und Pflege (StMGP) im Bayerischen Landesamt für Gesundheit und Lebensmittelsicherheit (LGL) ein Kommunalbüro für ärztliche Versorgung eingerichtet. Das Kommunalbüro analysiert als Kompetenz- zentrum im Auftrag des StMGP regionale Versorgungsstrukturen und berät Kommunen zur Verbesserung der ärztlichen Versorgung vor Ort. Anhand der Beratungsstatistik werden die kommunale Inanspruchnahme und die Anlässe des neuen flächendeckenden Beratungsangebots in Bayern im Zeitraum von 2012 bis 2015 beschrieben.

Ergebnisse Der Interventionsansatz des Kommunalbüros besteht aus den Komponenten Assessment, Fachberatung und Vernetzung sowie der Herausarbeitung der Handlungsoptionen und Gestaltungsmöglichkeiten im jeweiligen kommunalen Gesundheitsmanagement. Die Inanspruchnahme der Beratungsleistungen des Kommunalbüros in den zurückliegenden 4 Jahren war hoch: bis 31.11.2015 waren 233 Beratungsfälle und 155 intensive Beratungsfälle zu verzeichnen. Die hausärztliche Versorgung bildete mit $68 \%$ aller im Themengebiet der vertragsärztlichen Versorgung vorgebrachten Beratungsanlässe den inhaltlichen Schwerpunkt; 29\% betrafen die Allgemeine Fachärztliche Versorgung. Unter allen Anlässen bildeten die Themengebiete „Nachfolgesuche im Rahmen der Vorbereitung einer Übergabe einer Vertragsarztpraxis“ mit 33 \%, „Neuansiedlung von Vertragsärzten“ mit $23 \%$ sowie „Gründung einer Zweigpraxis“ mit 18\% die Interessensschwerpunkte.

Schlussfolgerung Das Kommunalbüro für ärztliche Versorgung bietet bayerischen Kommunen erstmals einen direkten Ansprechpartner von Landesseite für Fragen der ärztlichen Versorgung. Das Beratungsangebot wird sehr rege angenommen. Insbesondere die wohnortnahe hausärztliche Versorgung kristallisiert sich dabei als zentrales Thema auf kommunaler Ebene heraus. Die Einrichtung des Kommunalbüros für ärztliche Versorgung kann als objektives und neutrales Unterstützungsangebot für die kommunale Ebene und als Maßnahme zur Strukturverbesserung auf Landesebene Modellcharakter auch für andere Bundesländer haben.

\section{ABSTRACT}

Background Demographic changes pose serious challenges for the healthcare system. One important goal is to sustain the local healthcare provision in the future - especially in rural areas. In this context, more attention must be given to the statutory health service by communal as well as state authorities. Most of the municipalities have to tackle this problem for the first time and, due to lack of support, are faced with serious impediments. The aim of this paper is to describe the sphere of action of the Office for Medical Care in Districts and Municipalities (OMCDM) as well as its core outcome.

Methods The Bavarian Ministry of Health and Care (StMGP) established the OMCDM at the Bavarian Health and Food Safety Authority to support communal authorities. On behalf of the StMGP, this office analyses the existing set-ups of local health services and advises local authorities on improvement. For 2012-2015, the OMCDM database was analyzed for frequency and main reasons of contact with health services. 
Results The action of the OMCDM is driven by a comprehensive assessment, specialist counseling, and networking to develop action potential for the individual local health authorities. Over the past four years, there has been an increasing demand for support. Until 2015-11-30, 233 cases of counseling and 155 intensive counseling cases were recorded. The topics most frequently mentioned in these consultations were primary care by General Practitioners (68\%) and specialist care (29\%). Other important issues were the search for successors (33\%), the establishment of new practices ( $23 \%$ ) and opening of branch practices (18\%).

Conclusion On behalf of Bavarian government, the OMCDM offers easy access to objective and neutral counseling about statutory health services for Bavarian municipalities. This offer has been used frequently by districts and municipalities. Primary care by general practitioners on the local level was the most common reason to contact the OMCDM. The establishment of the Office for Medical Care in Districts and Municipality is a comprehensive support for local authorities and can be regarded as a pattern for other states in Germany.

\section{Einleitung}

Trotz einer sehr guten Ausgangslage steht die Gesundheitsversorgung in Deutschland vor vielfältigen Herausforderungen. Vor diesem Hintergrund sind neue Strategien, Modelle, Strukturen und Rahmenbedingungen für die Gesundheitsversorgung erforderlich [1-3]. Besonders diskutiert wird das Thema eines drohenden Fachkräftemangels [3]. Neben einem prognostizierten Mangel in den Pflegeberufen droht ein Ärztemangel - insbesondere hinsichtlich der hausärztlichen Versorgung in ländlichen, strukturschwachen Räumen [3-5]. In den letzten Jahren wurden auf Bundesebene mehrfach die gesetzlichen Rahmenbedingungen angepasst, um die wohnortnahe und flächendeckende vertragsärztliche Versorgung zu erhalten und weiterzuentwickeln ${ }^{1}$. Die Neufassung der Bedarfsplanungs-Richtlinie zum 1. Januar 2013 durch den Gemeinsamen Bundesausschuss zielte auf die Flexibilisierung, Weiterentwicklung und Verbesserung wohnortnaher Versorgung über z. B. die Definition von Versorgungsebenen, die Weiterentwicklung des Demografiefaktors sowie die Einbeziehung von Arztgruppen, die bisher nicht in die Bedarfsplanung einbezogen waren [6]. Außerdem ermöglicht die überarbeitete Richtlinie verstärkt regionale Entscheidungsspielräume bei der Bedarfsplanung.

Für die Sicherstellung, Verbesserung und Förderung der ambulanten ärztlichen Versorgung sind nach $\S 75,105$ SGB V grundsätzlich die Kassenärztlichen Vereinigungen zuständig. Aber auch auf kommunaler Ebene wird die Thematik der medizinischen Versorgung, besonders im ländlichen Raum, von politischen Mandatsträgern und Verwaltungen in den letzten Jahren vermehrt behandelt [7-9] und zunehmend v. a. hinsichtlich des Beitrags zur Schaffung geeigneter Rahmenbedingungen als „mögliches Handlungsfeld für die Kommunalpolitik“ ([10], S. 49) gesehen. Dies deckt sich mit Befragungsergebnissen, die andeuten, dass die Attraktivität eines Standortes und „weiche Standortfaktoren“ wie Rahmenbedingun-

\footnotetext{
${ }^{1} \mathrm{vgl}$. Gesetz zur Modernisierung der Gesetzlichen Krankenversicherung (GMG), Gesetz zur Änderung des Vertragsarztrechts und anderer Gesetze (VÄndG), Gesetz zur Stärkung des Wettbewerbs in der gesetzlichen Krankenversicherung (GKV-WSG), Gesetz zur Verbesserung der Versorgungsstrukturen in der gesetzlichen Krankenversicherung (GKV-VStG) sowie Gesetz zur Stärkung der Versorgung in der gesetzlichen Krankenversicherung (GKV-VSG)
}

gen für die Familie oder die Lebensqualität im Umfeld bei der Niederlassungsentscheidung von Ärzten eine zentrale Rolle spielen (vgl.: [11]).

Viele Kommunen befassen sich erstmals intensiv mit der aufgrund von Selbstverwaltung, Korporatismus und sektoraler Abgrenzung des deutschen Gesundheitssystems sehr komplexen Thematik. Sie stehen vor großen Herausforderungen. Zudem sind viele der Gemeinden sehr klein und haben geringe Personalressourcen. Bisher mangelte es an neutralen, qualitativ hochwertigen und auf den Einzelfall bezogenen Unterstützungs- und Beratungsangeboten für die kommunale Ebene. Für die Unterstützung der Kommunen wurde deshalb das Kommunalbüro für ärztliche Versorgung auf Grundlage der Regierungserklärung „Gesundheitsland Bayern - neue Strategien für eine menschliche Medizin“ (20.10.2011) vom Bayerischen Staatsministerium für Gesundheit und Pflege (StMGP) im Bayerischen Landesamt für Gesundheit und Lebensmittelsicherheit (LGL) in Nürnberg eingerichtet. Nach konzeptioneller Vorarbeit werden seit September 2012 die Dienstleistungen des Kommunalbüros angeboten.

Nachfolgend werden zunächst Ziele, Aufgaben, Zielgruppen und Interventionsansatz dieser deutschlandweit bisher einzigartigen Einrichtung vorgestellt. Darauf aufbauend werden Handlungsoptionen und Gestaltungsmöglichkeiten im kommunalen Gesundheitsmanagement diskutiert sowie die Inanspruchnahme und die Erfahrungen des Kommunalbüros berichtet. Schließlich wird ein kritisches Resümee für die Implementierungsphase gezogen.

\section{Ziele, Aufgabe und Zielgruppe des Kommunalbüros für ärztliche Versorgung}

Das Beratungs- und Unterstützungsangebot des Kommunalbüros für ärztliche Versorgung zielt darauf ab, Lösungsmöglichkeiten für Herausforderungen der künftigen gesundheitlichen Versorgung auf kommunaler Ebene gemeinsam mit Kommunen, der Kassenärztlichen Vereinigung Bayerns (KVB), Krankenhäusern und öffentlichem Gesundheitsdienst zu entwickeln - unbeschadet der gesetzlich normierten Sicherstellungsverpflichtung der Kassenärztlichen Vereinigung. Aufgabenschwerpunkt des Kommunalbüros ist es, als Kompetenzzentrum im Auftrag des StMGP die Verantwortlichen vor Ort im Sinne der „Hilfe zur Selbsthilfe“ bei der Weiterentwicklung der Gesundheitsversorgung zu unterstützen. Zielgruppe des Kommunalbüros für ärztliche Versorgung sind politische Mandatsträger und Verwaltungen der 3 kommunalen Ebenen in Bayern (Bezirke, Landkreise und Gemeinden) - insbesondere Bürgermeister von Gemeinden. Das Land Bayern besteht insgesamt aus 2056 politisch selbständigen Gemeinden, 96 Landkreisen und kreisfreien Städten sowie 7 Bezirken. Über eine Antragsberatung zum „Förderprogramm zum Erhalt und zur Verbesserung der ärztlichen Versorgung “ des Landes unterstützt das Kommunalbüro zudem die Weiterentwicklung der Gesundheitsversorgung durch Modellprojekte. 
- Tab. 1 Übersicht über 23 Indikatoren zu Demografie, Sozialstruktur, Gesundheit, Gesundheitsversorgung und Zukunftsperspektive.

\begin{tabular}{|c|c|}
\hline Indikatorengruppe & Indikatoren \\
\hline Rahmendaten (Demografie, Sozialstruktur, Gesundheit) & 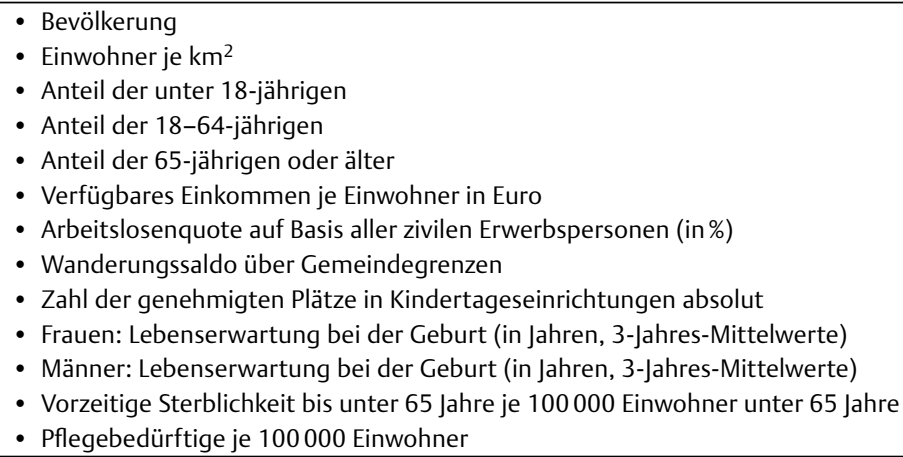 \\
\hline Gesundheitsversorgung (Struktur, Morbidität) & $\begin{array}{l}\text { - Einwohner je Arzt in ambulanten Einrichtungen } \\
\text { - Einwohner je Hausarzt } \\
\text { - Hausärzte: Versorgungsgrad (in \%) } \\
\text { - Anzahl Krankenhäuser } \\
\text { - Krankenhausbettendichte je } 1000 \text { Einwohner } \\
\text { - Entfernung zum nächsten Krankenhaus (in km)a } \\
\text { - Bösartige Neubildungen je } 100000 \text { Einwohner, altersstandardisiert } \\
\text { - Krankenhausfälle je } 100000 \text { Einwohner } \\
\end{array}$ \\
\hline Zukunftsperspektive & $\begin{array}{l}\text { - Bevölkerungsveränderung (in \%) } \\
\text { - Hausärzte: Anteil } 60 \text { Jahre und älter (in \%) }\end{array}$ \\
\hline
\end{tabular}

\section{Interventionsansatz}

\section{Assessment}

Als Basis der Arbeit des Kommunalbüros für ärztliche Versorgung dient ein systematisches und objektives Assessment zur Identifikation der Ausgangslage im Sinne quantitativer und qualitativer Erhebung und Analyse der jeweiligen regionalen Versorgungsstrukturen. Das Assessment wird durch das Kommunalbüro unter Einbeziehung der Verantwortlichen vor Ort (z. B. Bürgermeister, Kommunalverwaltungen, niedergelassene Ärzte, weitere Leistungserbringer) durchgeführt. Eine Grundlage der regionalen Analysen bilden 23 Indikatoren zu Demografie, Sozialstruktur, Gesundheit, Gesundheitsversorgung und Zukunftsperspektive ( Tab. 1). Das Monitoring der Gesundheitsindikatoren wird ergänzt durch Befunde aus Gesprächen, Vor-Ort-Begutachtungen von Strukturen und Prozessen sowie der qualitativen Auswertung von Dokumenten (z. B. Informationsmaterialien der Gemeinde oder von Leistungserbringern), die das Kommunalbüro durchführt. Wenn bspw. die Übergabe einer Hausarztpraxis geplant ist, dann werden Stärken und Schwächen der Organisation, Struktur und (Personal-)Ausstattung der Praxis, sowie die Attraktivität des Praxisstandortes (u. a. Lage, Infrastruktur, Umfeld) analysiert. Zudem werden gegebenenfalls bereits eingeleitete Aktivitäten erhoben und in Bezug auf Erreichtes und zu erwartende Erfolgsaussichten ausgewertet.

\section{Fachberatung und Vernetzung}

Aufbauend auf der Analyse finden ausführliche Beratungsgespräche - in der Regel vor Ort - statt. Zu Beginn stehen dabei zunächst die Vermittlung relevanter Informationen über Hintergründe, Strukturen und Ansprechpartner der Versorgung sowie präventive Handlungsstrategien im Vordergrund. Zentrale Themen sind z. B. die vertragsärztliche Bedarfsplanung, die jeweils aktuelle Versor- gungssituation, Zuständigkeiten oder mögliche Organisationsformen von Arztpraxen. Zudem ist die Sensibilisierung für und Schärfung des Problembewusstseins, z. B. für aktuelle Trends in der Versorgung und daraus abzuleitende Entwicklungen und Herausforderungen fester Bestandteil der Beratungsaktivitäten. Gleiches gilt für die Entwicklung individueller und maßgeschneiderter Lösungsoptionen bei Problemen zur Verbesserung der Versorgungssituation. Ziel ist jeweils die Entwicklung einer passgenauen Strategie mit Festlegung konkreter Schritte und zeitlicher Abläufe sowie die Begleitung, Bewertung und ggf. Weiterentwicklung oder Anpassung entsprechender Umsetzungsprozesse durch das Kommunalbüro für ärztliche Versorgung. Bspw. kann ein erster Schritt darin bestehen, vertrauliche Gespräche mit dem/den vor Ort niedergelassenem/n Hausarzt/Hausärzten zu führen, um Details zu dessen Ideen und Zeitläufen einer anstehenden Praxisübergabe zu erfahren. Aufbauend auf den Gesprächen kann erneut mit dem Kommunalbüro gesprochen werden, um die Gesprächsergebnisse zu bewerten und die nächsten konkreten Schritte zu planen (z. B. das gemeinsame Werben von Kommune und Arzt um einen Nachfolger inkl. der Erstellung einer geeigneten Kommunikationsstrategie mit Inhalten, Form und Verbreitungswegen).

Die Unterstützungsleistungen des Kommunalbüros sind Prozess begleitend und erstrecken sich in der Regel über längere, nicht selten mehrjährige Zeiträume. Sie orientieren sich konsequent am jeweiligen Bedarf vor Ort. Das Angebot des Kommunalbüros basiert auf den Prinzipien Objektivität, Neutralität, Systematik, Einzelfallbezug, Kompetenz und Gemeinwohlorientierung. Es steht für die Kommunen landesweit kostenfrei zur Verfügung.Das Kommunalbüro setzt auf die Beteiligung und Integration der jeweils relevanten Akteure aus Politik und Verwaltung sowie der Leistungserbringer. Es wirkt darauf hin, dass im Sinne eines konzertierten Vorgehens zuständige Ressorts und Personen in die Entwicklung lösungsorientierter potentieller Handlungsstrategien einbezogen 
werden. Insbesondere für ländliche Gemeinden bietet es sich zudem an, sich mit Nachbargemeinden auf ein abgestimmtes Vorgehen zu verständigen, nach gemeindeübergreifenden Lösungen im Sinne regionaler Ansiedlungsförderung und Attraktivität zu suchen und (kostenintensive) Doppelstrukturen oder -aktivitäten zu vermeiden. Das Kommunalbüro unterstützt die Verantwortlichen vor Ort deshalb bei der Weiterentwicklung und Verbesserung gemeindenaher Vernetzungsaktivitäten in der Gesundheitsversorgung. Die unteren Behörden für Gesundheit wirken dabei idealerweise als fachkundige Stellen sowohl bei der Beobachtung und Analyse als auch in der Politikberatung und gegebenenfalls bei der Maßnahmenentwicklung vor Ort im Sinne gesundheitspolitischer Steuerung mit. Um die regionalen Versorgungsstrukturen besser zu koordinieren und eine nachhaltige, regionale Förderungsstruktur zu implementieren, können „Regionale Gesundheitskonferenzen“ auf Kreisebene dienlich sein. Durch die Einbindung von stationären Leistungserbringern haben diese das Potenzial, sowohl einer verbesserten regionalen als auch sektorenübergreifenden Kooperation zwischen ambulantem und stationärem Sektor wichtige Impulse zu geben [12-14]. Das Kommunalbüro empfiehlt deshalb entsprechende Strukturbildungen auf Kreisebene - bspw. Gesundheitsregionen plus2 [13] - und wirkt bei Gesundheitskonferenzen mit.

\section{Transfer}

Über die Analyse, Sensibilisierung, Beratung und Unterstützung im Einzelfall hinaus, führt das Kommunalbüro für ärztliche Versorgung die in einzelnen Kommunen entwickelten Ansätze systematisch zusammen und leitet daraus allgemeingültige Empfehlungen für Lösungsstrategien ab. Fester Bestandteil der Arbeit des Kommunalbüros für ärztliche Versorgung ist auch die fortlaufende Analyse aktueller Problemlagen, Forschungsergebnisse und gesetzlicher Rahmenbedingungen des Gesundheitssystems und der Versorgungspraxis sowie deren Verbreitung (z. B. über Informationsmaterialien oder Veranstaltungen wie Versorgungskonferenzen, Bürgermeisterdienstbesprechungen, Arbeitskreissitzungen und Workshops). Hierzu finden u. a. regelmäßig Fachgespräche mit dem StMGP, kommunalen Verantwortungsträgern, Akteuren der Gesundheitsversorgung und weiteren Fachleuten statt. Das Kommunalbüro für ärztliche Versorgung versteht sich in diesem Zusammenhang zudem als Dienstleister für die beratenen Kommunen, indem es aktuelle Entwicklungen auf den einzelnen Beratungsfall bezogen auswertet und entsprechende Konsequenzen und Handlungsbedarfe oder (neu eröffnete) Handlungsoptionen ableitet und zeitnah kommuniziert. So können sich bspw. durch neue gesetzli-

\footnotetext{
2Die oberste Zielsetzung der 2015 vom StMGP eingeführten und geförderten, sogenannten „Gesundheitsregionen plus“ ist es, den Gesundheitszustand der Bevölkerung in Bayern zu verbessern und die gesundheitsbezogene Lebensqualität zu erhöhen. Als ein fachlich kompetentes Netzwerk regionaler Akteure des Gesundheitswesens bemühen sich die Gesundheitsregionenplus um die Optimierung der regionalen Gesundheitsvorsorge und -versorgung. Bestehend aus einem Gesundheitsforum mit Management- und Steuerungsaufgaben, themenbezogenen Arbeitsgruppen und einer koordinierenden Geschäftsstelle, sollen sich die Gesundheitsregionen plus vorrangig den Handlungsfeldern Gesundheitsförderung/Prävention sowie der Gesundheitsversorgung widmen.
}

che Regelungen oder die Fortschreibung der Bedarfsplanung (z. B. sich ändernde Versorgungsgrade oder Zuschnitte von Planungsbereichen) zusätzliche Möglichkeiten ergeben, oder es müssen bereits entwickelte Strategien angepasst werden. Die Transferaktivitäten dienen zudem der Vernetzung und sie leisten einen wichtigen Beitrag zur Förderung des Informationsflusses und des Know-how-Transfers zwischen Landes-, Bezirks- und regionaler Ebene sowie zwischen Wissenschaft und Praxis.

\section{Handlungsoptionen und Gestaltungs- möglichkeiten im kommunalen Gesundheits- management}

Wegen Zuständigkeitsbereichen von kommunaler Ebene auf der einen und Selbstverwaltungsorganen sowie Leistungserbringern auf der anderen Seite sind ein erfolgversprechendes kommunales Gesundheitsmanagement, ein gelingendes Zusammenwirken und die enge Abstimmung des Vorgehens wichtig. Bei der Suche nach einem Nachfolger für einen frei werdenden Kassensitz bspw. bilden Gemeinde, Landkreis, Region und die Arztpraxis eine „eng verwobene“ Einheit von (günstigen oder weniger günstigen) Standortfaktoren.

\section{Handlungsoptionen der Kommunen}

Handlungsoptionen der kommunalen Hand liegen dabei zuständigkeitshalber insbesondere in der Gestaltung und Beeinflussung von Rahmenbedingungen und Lebensqualität im Wohn- und Arbeitsumfeld. Denn diese scheinen die Niederlassungsentscheidung von Ärzten stark zu beeinflussen. So verdichten sich die Hinweise darauf, dass die sogenannten „weichen Standortfaktoren“ durchaus „harte“ Einflussgrößen auf Niederlassungsentscheidungen darstellen [11]. Die kommunale Hand hat demnach über die Gestaltung von Standortfaktoren durchaus relevante Einflussmöglichkeiten auf die Weiterentwicklung lokaler und regionaler Gesundheitsversorgung. Das Kommunalbüro sensibilisiert die beratenen Kommunen hinsichtlich diesbezüglicher zentraler Standortfaktoren wie Betreuungs- und Schulangebote für die Kinder, berufliche Möglichkeiten für den Lebenspartner, Wohnqualität, Freizeitmöglichkeiten, kulturelles Angebot, infrastrukturelle Anbindung (inkl. des ÖPNV) sowie - bei Bedarf - die Bereitstellung mietgünstiger Praxisräume oder geeigneten Baulandes und die Unterstützung bei der Suche nach Investoren. Bei Bedarf können Kommunen darüber hinaus - auch gemeindeübergreifend - kooperative Niederlassungsoptionen und familienfreundliche Formen der Berufsausübung oder Kooperationen fördern sowie Hol- und Bringdienste für Patienten unterstützen [11]. Das Kommunalbüro unterstützt die Kommunen bei der Analyse der Stärken und Schwächen und der Entwicklung einer geeigneten Strategie zur Steigerung der Attraktivität des Standortes. Die angesprochenen Handlungsoptionen zeigen, dass Fragen des Gesundheitsmanagements mehrere Zuständigkeiten berühren und ein konzertiertes Vorgehen erfordern. Idealerweise arbeiten dazu unterschiedliche Ressorts und Sektoren wie Gesundheit und Soziales, Bildung und Wissenschaft, Bauen und Verkehr sowie Kultur und Sport zusammen. 


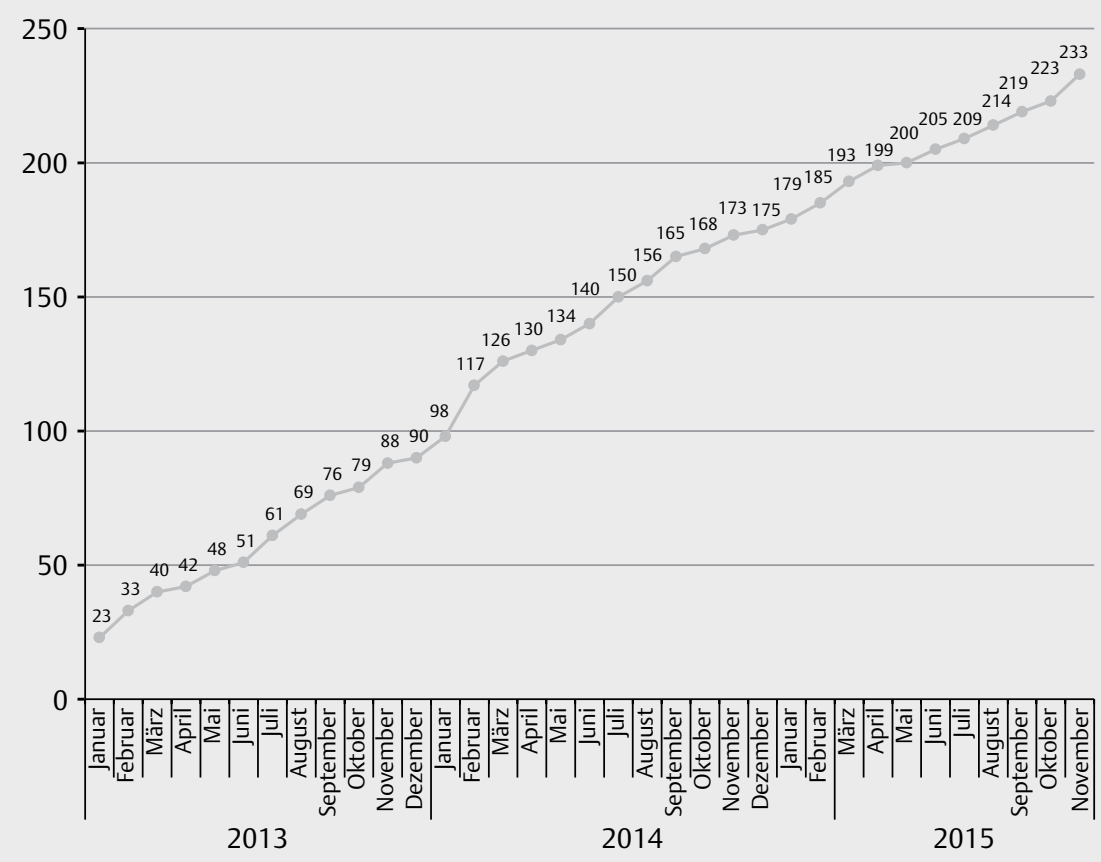

> Abb. 1 Beratungsfälle des Kommunalbüros für ärztliche Versorgung (kumuliert) vom 1.1.2013 bis 30.11.2015.

\section{Handlungsoptionen der Leistungserbringer}

Neben Rahmenbedingungen und Lebensqualität haben bekanntermaßen die Ausstattung von Arztpraxen sowie ihre Struktur und Organisation nicht unerhebliche Auswirkungen auf die Attraktivität und Niederlassungsfreundlichkeit [15]. Das Kommunalbüro informiert und sensibilisiert die am Beratungsprozess beteiligten Ärzte deshalb bspw. hinsichtlich veränderter beruflicher Rollenvorstellungen von Ärzten: So kommt der Vereinbarkeit von Familie und Beruf, der Etablierung familienfreundlicher (Arbeits-)Konzepte sowie der Work-Life-Balance eine immer stärkere Bedeutung zu [16-19]. In der Folge werden kooperative, flexible und an der jeweiligen Lebensphase orientierte Formen der Praxisorganisation wie Praxisgemeinschaften, Berufsausübungsgemeinschaften oder Medizinische Versorgungszentren erheblich stärker als in der Vergangenheit nachgefragt $[18,20]$. Mittlerweile entscheiden sich 46,2\% der hausärztlichen Existenzgründer für eine Kooperation. Der Einstieg in eine Berufsausübungsgemeinschaft ist dabei die häufigste Kooperationsform [21]. Gleiches gilt für Möglichkeiten als angestellter Arzt im ambulanten Bereich tätig zu sein [19, 22, 23]. Kooperative Organisationsformen bieten darüber hinaus häufig auch wirtschaftliche Vorteile, sie begünstigen den fachlichen Austausch [24] und können zu einer Steigerung der Arbeitszufriedenheit beitragen [25]. Im Beratungsprozess werden diese Aspekte bspw. mit dem Praxis abgebenden Arzt vor Ort gemeinsam diskutiert und mit der jeweiligen Praxissituation abgeglichen. Darauf aufbauend werden konkrete Möglichkeiten und Grenzen der Weiterentwicklung der Praxis im Sinne der Attraktivitätssteigerung eruiert und wenn möglich in konkrete Handlungsstrategien überführt. Neben den organisationsbezogenen Aspekten entscheiden auch „ausstattungsbezogene“ Kriterien über den Attraktivitätsgrad bestehender Arztpraxen: Weist die zur Übergabe stehende Praxis einen Investitionsstau auf? Kann die Organisation der Praxis noch optimiert werden? [26, 27] Ist die Praxis barrie- refrei ausgestattet oder zugänglich? [27, 28] Auch die Qualifikation des Praxispersonals gilt es in den Blick zu nehmen. Abgabewillige Ärzte sind demnach aufgerufen, rechtzeitig entsprechende Aspekte kritisch zu hinterfragen und ggf. eine Kurskorrektur einzuleiten, um die Attraktivität der Praxis günstig zu beeinflussen [15]. Nicht zuletzt gilt es für den Praxis abgebenden Arzt in einem „klassischen Käufermarkt“ [15] auch die eigenen finanziellen Forderungen kritisch zu hinterfragen und wenn nötig zu korrigieren. Das Kommunalbüro moderiert die entsprechenden Prozesse und gibt - wenn nötig - Unterstützung, z. B. durch Nennung geeigneter Ansprechpartner für Ärzte.

Selbstverständlich stellen die vorangegangenen Handlungsoptionen und -felder nur eine Auswahl von Möglichkeiten dar. Es ist jeweils im Einzelfall - nach eingehender Analyse von Ausgangslage, Rahmenbedingungen, Möglichkeiten und Zielen - in Abstimmung mit den jeweils handelnden Akteuren und unter Berücksichtigung der bestehenden Rahmenbedingungen, wie sie bspw. durch die Bedarfsplanung, aber auch kommunal- und wettbewerbsrechtliche Regelungen vorgegeben sind, nach passgenauen und zielführenden Lösungswegen zu suchen. Idealerweise greifen die Aktivitäten der kommunalen Hand und der (regionalen) Leistungserbringer bei der Vorbereitung einer Praxisübergabe oder dem Bemühen um eine Neuansiedlung abgestimmt ineinander, um die Attraktivität des Standortes zu erhalten, weiterzuentwickeln und nach außen zu bewerben.

\section{Inanspruchnahme des Kommunalbüros und Beratungsanlässe}

Das Kommunalbüro wurde in den zurückliegenden gut dreieinhalb Jahren sehr gut nachgefragt: Bis 30.11.2015 waren 233 Beratungsfälle und 155 intensive Beratungsfälle zu verzeichnen. > Abb. 1 
zeigt die dynamisch gestiegene Anzahl der Beratungsfälle vom 1.1.2013 bis 30.11.2015.

$68 \%$ aller im Rahmen der intensiven Beratungsfälle vorgebrachten Anlässe im Themengebiet der vertragsärztlichen Versorgung betrafen die hausärztliche Versorgung, 29\% die „Allgemeine Fachärztliche Versorgung“. Die „Spezialisierte Fachärztliche Versorgung“ hingegen war mit 3 \% nur selten, die „Gesonderte Fachärztliche Versorgung“ bisher überhaupt kein Anlass in der Beratung. Eine detailliertere Auswertung aller Beratungsanlässe der Intensivberatungen ergibt, dass die Themengebiete „Nachfolgesuche im Rahmen der Vorbereitung einer Übergabe einer Vertragsarztpraxis“ mit 33 \%, „Neuansiedlung von Vertragsärzten“ mit 23 \% sowie „Gründung einer Zweigpraxis“ mit $18 \%$ die Interessensschwerpunkte bildeten. Weitere Anlässe betrafen die Themengebiete „Ärztehäuser/medizinische Versorgungszentren/Berufsausübungsgemeinschaften“ (14\%), Bereitschaftsdienst (2\%) sowie „Sonstiges“ ( $9 \%)$, wie stationäre, pflegerische und pharmazeutische Versorgung. Es kann davon ausgegangen werden, dass die Anlässe die wahrgenommenen Herausforderungen, den empfundenen „Leidensdruck" und die Art der Probleme auf kommunaler Ebene wiederspiegeln.

Aufgrund der relativ langen Zeitläufe - z. B. im Kontext der Vorbereitung und Abwicklung der Übergabe von Arztpraxen - gestalten sich die Beratungsprozesse langfristiger. Sie erstrecken sich in der Regel über mehrere Jahre. Von den 155 intensiven Beratungsfällen wurden bis Ende November 201534 abgeschlossen, da auf Nachfrage des Kommunalbüros kein weiterer Beratungs- und Unterstützungsbedarf seitens der beratenen Kommune mehr geäußert wurde. In 19 Fällen konnten bereits konkrete Lösungen gefunden werden: So konnte bspw. in 11 Fällen die Nachfolge einer bestehenden Praxis geregelt werden, in 4 Fällen konnten Hausarztpraxen erfolgreich neu angesiedelt werden und bei 3 Fällen konnte über die Etablierung einer Filialpraxis die Versorgung weiterentwickelt werden. Zudem konnten (häufig zusätzlich) neue Strukturen erfolgreich etabliert werden, indem z. B. Ärztehäuser oder überörtliche Zusammenschlüsse realisiert wurden.

In 13 Fällen wurde der Beratungsfall abgeschlossen, weil sich die Kommunen sehr gut beraten fühlten, um anstehende Aktivitäten selbstständig einzuleiten und weiter zu betreiben. Hier ist dem Kommunalbüro für ärztliche Versorgung nicht bekannt, ob und mit welchem Ergebnis erarbeitete Strategien zwischenzeitlich umgesetzt werden konnten. Zum Teil handelt es sich dabei auch um langfristig angelegte, „präventive“ Strategien zur Weiterentwicklung der Gesundheitsversorgung ohne akuten Handlungsdruck vor Ort.

In 2 der abgeschlossenen Beratungsfälle zeigten sich die beratenen Kommunen unzufrieden mit den prinzipiell bestehenden Lösungsmöglichkeiten vor Ort (z. B. aufgrund bestehender „Restriktionen“ durch die Bedarfsplanung). In beiden Fällen wurde jedoch unabhängig davon betont, dass die Beratungsleistungen des Kommunalbüros sehr positiv bewertet werden.

\section{Resümee}

Mit dem Kommunalbüro haben die Kommunen in Bayern erstmals einen direkten Ansprechpartner für Fragen der ärztlichen Versorgung von Landesseite erhalten. Das Beratungsangebot wird stark nachgefragt. Zentrales Thema ist dabei die wohnortnahe hausärztliche Versorgung. Ergebnisse bisher abgeschlossener Beratungsfälle weisen darauf hin, dass die empfohlenen Strategien erfolgreich sein können.

Die Einrichtung des Kommunalbüros kann als Unterstützungsangebot für die kommunale Ebene und als Maßnahme zur Strukturverbesserung auf Landesebene Modellcharakter auch für andere Bundesländer haben. Das Fachkonzept scheint geeignet, dabei zu unterstützen, vorhandene Gestaltungsmöglichkeiten zur Verbesserung der gesundheitlichen Versorgung zu erkennen und zielgerichtet wahrzunehmen. Empfohlen wird diesbezüglich eine Anbindung an den öffentlichen Gesundheitsdienst auf Landesebene oder - falls nicht mit entsprechendem Zuschnitt vorhanden - an die Landesvereinigungen oder Landeszentralen für Gesundheit sowie eine Vernetzung mit bestehenden Strukturen wie regionalen oder Landesgesundheitskonferenzen.

Die Zusammenarbeit mit der für die Sicherstellung zuständigen KVB hat sich als fruchtbar für die Beratungsprozesse herausgestellt. Dies gilt auch für die Kooperation mit dem öffentlichen Gesundheitsdienst. Die Aktivitäten sind darüber hinaus in hohem Maße anschlussfähig an die kommunale Gesundheitsberichterstattung und Gesundheitszieleprozesse des öffentlichen Gesundheitsdienstes auf kommunaler Ebene. Über die Beteiligung an Versorgungskonferenzen auf Bezirksebene wird zudem ein Beitrag zur Verzahnung zwischen Land und kommunaler Ebene geleistet. Die Verbindung mit Förderprogrammen des Landes eröffnet die Möglichkeit Gesundheitsversorgung durch Modellprojekte weiterzuentwickeln oder regionale Vernetzungsaktivitäten, wie Gesundheitsregionenplus, zu flankieren.

\section{Interessenkonflikt}

Die Autoren sind am Bayerischen Landesamt für Gesundheit und Lebensmittelsicherheit tätig, in dem das Kommunalbüro für ärztliche Versorgung angesiedelt ist.

\section{Literatur}

[1] Sachverständigenrat zur Begutachtung der Entwicklung im Gesundheitswesen (SVR). Koordination und Integration - Gesundheitsversorgung in einer Gesellschaft des längeren Lebens: Sondergutachten 2009. Berlin: Nomos; 2010

[2] Sachverständigenrat zur Begutachtung der Entwicklung im Gesundheitswesen (SVR). Wettbewerb an der Schnittstelle zwischen ambulanter und stationärer Gesundheitsversorgung: Sondergutachten 2012. Bern: Huber; 2012

[3] Sachverständigenrat zur Begutachtung der Entwicklung im Gesundheitswesen (SVR). Bedarfsgerechte Versorgung - Perspektiven für ländliche Regionen und ausgewählte Leistungsbereiche. Bonn/Berlin: 2014

[4] Knieps F, Amelung VE, Wolf S. Die Gesundheitsversorgung in schwer zu versorgenden Regionen - Grundlagen, Definition, Problemanalyse. Gesundheits- und Sozialpolitik 2012; 85: 8-19

[5] Kopetsch T. Dem deutschen Gesundheitswesen gehen die Ärzte aus! Studie zur Altersstruktur- und Arztzahlentwicklung. Berlin: Bundesärztekammer und Kassenärztliche Bundesvereinigung; 2010 
[6] Gemeinsamer Bundesausschuss. Tragende Gründe zum Beschluss des Gemeinsamen Bundesausschusses über eine Neufassung der Bedarfsplanungs-Richtlinie: Bedarfsplanung gemäß GKV-VStG. Berlin: 2012

[7] Deutscher Landkreistag. Rolle der Landkreise in der gesundheitlichen Versorgung - Positionen des Deutschen Landkreistages in der Gesundheitspolitik. Berlin: 2013

[8] Deutscher Landkreistag. Medizinische Versorgung im ländlichen Raum. Erwartungen des Deutschen Landkreistages an die Gestaltung politischer Rahmenbedingungen für die medizinische Versorgung außerhalb der Ballungsräume. Berlin: 2010

[9] Landsberg G. Positionspapier „Ärztliche Versorgung im ländlichen Raum“. Berlin: 2010

[10] Süssmuth A. Sicherstellung der Gesundheitsversorgung im ländlichen Raum. Handlungsfelder und Chancen der Kommunalpolitik. München: AVM; 2013

[11] Langer A, Ewert T, Hollederer A et al. Literaturüberblick über niederlassungsfördernde und -hemmende Faktoren bei Ärzten in Deutschland und daraus abgeleitete Handlungsoptionen für Kommunen. Gesundheitsökonomie \& Qualitätsmanagement 2014; 20 : $11-18$

[12] Hollederer A. Gesundheitskonferenzen in Deutschland: ein Überblick. Das Gesundheitswesen 2015; 77: 161-167

[13] Hollederer A, Eicher A, Stühler K et al. Vernetzung, Koordination und Verantwortung durch Gesundheitsregionenplus: Neue gesundheitspolitische Ansätze und Entwicklungen in Bayern. Gesundheitswesen 2015; ohne Seitenzahl, DOI: 10.1055/s-0035-1555892

[14] Hollederer A, Stühler K. Kooperation im Gesundheitswesen: Formative Evaluation des Modellprojekts Regionale Gesundheitskonferenzen in Bayern. Gesundheitswesen 2016; ohne Seitenzahl, DOI: 10.1055/s0041-110673

[15] Kirschner G, Wigge P. Arzt und Praxisabgabe. Köln: Deutscher Ärzte-Verlag; 2014

[16] Bühren A. Einleitung. In: Bühren A, Schoeller AE (Hrsg.). Familienfreundlicher Arbeitsplatz für Ärztinnen und Ärzte. Berlin: Bundesärztekammer; 2010: 11-14
[17] Kassenärztliche Vereinigung Bayerns. Ärztinnen in der vertragsärztlichen Versorgung. München: 2012

[18] Hartmannbund - Verband der Ärzte Deutschlands. Positionspapier „Zukunft des Arztberufes“. Herausforderungen und Perspektiven. Lösungsansätze aus der Umfrage unter den Medizinstudierenden des Hartmannbundes. Berlin: 2012

[19] Fuchs C, Koch T, Scriba PC. Perspektiven junger Ärztinnen und Ärzte in der Patientenversorgung. Köln: Deutscher Ärzte-Verlag; 2013

[20] Bühren A, Schoeller AE. Familienfreundlicher Arbeitsplatz für Ärztinnen und Ärzte. Lebensqualität in der Berufsausübung. Berlin: Bundesärztekammer; 2010

[21] Zentralinstitut für die kassenärztliche Versorgung. Existenzgründungsanalyse für Hausärzte 2012 - Eine Analyse des Zentralinstituts der kassenärztlichen Versorgung und der Deutschen Apotheker- und Ärztebank. Berlin: 2014

[22] Bundesärztekammer. Die ärztliche Versorgung in der Bundesrepublik Deutschland. Ergebnisse der Ärztestatistik zum 31.12.2011. Berlin: 2012

[23] Osterloh F. Mehr Ärztinnen, mehr Angestellte. Deutsches Ärzteblatt 2014; 111: 672-673

[24] Binsch H, Frehse M, Kleinke S et al. Welche Organisationsformen stehen für Kooperationen zur Wahl? In: Kirschner G, Frehse M (Hrsg.). Arzt und Niederlassung. Köln: Deutscher Ärzte-Verlag; 2013

[25] Erler A, Beyer M, Welbers G et al. Zusammenschluss von Hausarztpraxen zum SCHAAZ1 - Auswirkungen auf Arbeitszufriedenheit und Burnout-Risiko. Zeitschrift für Allgemeinmedizin 2012; 88: 303-312

[26] Klapp E. Abgabe und Übernahme einer Arztpraxis. Berlin: Springer; 2006

[27] Stiller TC. Übernahme und Gründung einer Arztpraxis. Berlin: Springer; 2013

[28] Kassenärztliche Bundesvereinigung. Barrieren abbauen. Ideen und Vorschläge für Ihre Praxis. Berlin: 2013 